\title{
Reduced susceptibility to daptomycin in methicillin- resistant Staphylococcus aureus isolated from catheter-related bloodstream infections
}

\section{Sho Ohyatsu}

Teikyo University Mizonokuchi Hospital

\section{Tomoyuki Nariyama}

Teikyo University Mizonokuchi Hospital

\section{Kotaro Matsumoto}

Teikyo University Mizonokuchi Hospital

\section{Yuki Moritoki}

Akita University Graduate School of Medicine

Kentaro Kikuchi ( $\nabla$ kentaro@med.teikyo-u.ac.jp )

Teikyo University Mizonokuchi Hospital https://orcid.org/0000-0002-2263-0711

\section{Research}

Keywords: daptomycin, susceptibility, methicillin-resistant Staphylococcus aureus, catheter-related bloodstream infection, biofilm

Posted Date: October 28th, 2021

DOI: https://doi.org/10.21203/rs.3.rs-970680/v1

License: (c) (1) This work is licensed under a Creative Commons Attribution 4.0 International License. Read Full License 


\section{Abstract \\ Background}

The appearance of reduced susceptibility to daptomycin in methicillin-resistant Staphylococcus aureus (MRSA) has recently been reported. It is unclear how likely MRSA involved in catheter-related bloodstream infections (CRBSI) is to dampen susceptibility to daptomycin. We investigated the minimum inhibitory concentrations (MIC) of daptomycin in MRSA isolated from the blood of patients with CRBSI and examined how it was affected by previous anti-MRSA drug treatment.

\section{Methods}

A total of 115 patients whose blood culture samples were found to contain MRSA were enrolled in this study. The MIC of daptomycin and vancomycin and whether the subjects had a history of anti-MRSA drug treatment were investigated and compared between the CRBSI and non-CRBSI groups.

\section{Results}

The mean MIC of daptomycin was significantly higher for the 46 CRBSI-related MRSA isolates than for the 69 non-CRBSI-related MRSA isolates ( 0.78 vs. 0.33 , respectively; $p<0.0001)$. Among the CRBSI-related MRSA isolates, those collected from patients with a history of anti-MRSA drug treatment had significantly higher MIC (1.27 vs. 0.53 , respectively; $p<0.01)$. During treatment, MRSA was detected again in $10 \mathrm{CRBSI}$ and 4 non-CRBSI patients, and all of the CRBSI-related MRSA isolates exhibited 1-2 log2 increases in their daptomycin MIC.

\section{Conclusions}

It is considered that when MRSA in catheter biofilms is exposed to anti-MRSA drugs, strains with reduced susceptibility to daptomycin are able to survive and disperse into the blood. Catheters should be removed if an MRSA-induced CRBSI is suspected. Further study of whether high-dose daptomycin treatment is effective when catheters cannot be immediately removed is needed.

\section{Background}

Most of the causative bacteria of catheter-related bloodstream infections (CRBSI) belong to the genus Staphylococcus, and they are often methicillin-resistant [1]. Therefore, vancomycin is often selected as an initial treatment when a CRBSI is suspected. However, it should be noted that there have been reports of nosocomial infections of vancomycin-resistant Enterococcus [2], and the frequency of heterogeneous vancomycin-intermediate $S$. aureus among methicillin-resistant Staphylococcus aureus (MRSA) has 
increased in Japan [3]. Also, biofilms form on MRSA-colonized catheters, and vancomycin has low biofilm permeability [4].

Therefore, daptomycin was developed as an anti-MRSA drug with high biofilm permeability. Daptomycin binds to bacterial cell membranes, causes membrane depolarization, and inhibits DNA and RNA synthesis by inducing the efflux of potassium ions [5]. Ten years have passed since it was first used in Japan, and MRSA that have reduced susceptibility to daptomycin have unexpectedly been reported. Such bacteria have been detected in cases involving bloodstream infections [6], endocarditis [7], and osteomyelitis [8], and the patients in these cases were previously administered anti-MRSA drugs.

To date, the risk of the appearance of reduced susceptibility to daptomycin in CRBSI-related MRSA is unclear. In this study, we investigated the minimum inhibitory concentrations (MIC) of daptomycin in MRSA isolated from CRBSI patients and examined how it was affected by previous anti-MRSA drug treatment. Moreover, we considered the countermeasures that should be taken in the clinical setting.

\section{Methods}

\section{Patients}

A total of 115 patients who were treated at our hospital or related facilities during the four years from May 2017 to April 2021 and whose blood culture samples were found to contain MRSA were enrolled in this study. The MIC of daptomycin and vancomycin and whether the subjects had a history of anti-MRSA drug treatment were investigated and compared between the CRBSI and non-CRBSI groups. A CRBSI was defined as when a pathogen was detected in one or more blood cultures, and the detected pathogen was only associated with infection foci located in a central venous catheter [9], and the same bacterium was detected in catheter tip cultures and peripheral blood cultures.

\section{Blood Cultures}

Two sets of blood culture bottles containing BD BACTEC 23F (for aerobic cultures) or 22F (for anaerobic cultures) (Becton Dickinson and Co. Franklin Lakes, NJ, USA) in a BD BACTEC smart container GT (Becton Dickinson and Co.) were used. Bottles that had been infused with a patient's blood were immediately attached to the BD BACTEC FX system (Becton Dickinson and Co.), and culturing was performed at $35{ }^{\circ} \mathrm{C}$ for 5 days. Bacterial identification and drug susceptibility testing were performed using the MicroScan Walkaway 96 plus system (Beckman Coulter Inc., Brea, CA, USA) and the Pos Combo $1 \mathrm{~J}$ panel.

\section{Catheter Tip Cultures}


The catheter tip cultures were performed according to the quantification method developed by BrunBuisson et al [10]. Namely, a 5-cm catheter tip was placed in a 15-mL tube, soaked in $1 \mathrm{~mL}$ of trypticase soy broth, and vortexed. Ten $\mu \mathrm{L}$ of the resultant solution was inoculated into an agar plate, which was then cultured at $35^{\circ} \mathrm{C}$ for 2 days. When $\geq 10^{3}$ colony-forming units $/ \mathrm{mL}$ were observed, the infection was judged to be significant, and bacterial species identification and drug susceptibility testing were performed in the same manner as for the blood cultures.

\section{Statistical analysis}

GraphPad Prism version 9.0 for Macintosh (GraphPad Software, San Diego, CA, USA) was used for the statistical analyses. Fisher's exact test was used for comparisons of the frequency of previous anti-MRSA drug treatment. The non-parametric Mann-Whitney test and multiple regression analysis were used for comparisons of MIC values. P-values of $<0.05$ were regarded as significant.

\section{Results}

\section{Characteristics of the patients}

Of the 115 cases in which MRSA was detected in blood cultures, 46 involved CRBSI, and 69 did not. There was no difference in mean age or sex distribution between the CRBSI and non-CRBSI groups $(\mathrm{p}=0.06$ and $p=0.08$, respectively) (Table 1 ). Sixteen patients with CRBSI and 5 of the non-CRBSI patients had a history of anti-MRSA drug treatment $(p=0.0003)$.

\section{The Minimum Inhibitory Concentrations Of Daptomycin And Vancomycin}

The mean MIC of daptomycin was $0.78 \pm 0.84$ for the CRBSI-related MRSA isolates, which was significantly higher than that for the non-CRBSI-related MRSA isolates $(0.33 \pm 0.17, p<0.0001)$ (Figure 1a). The mean MIC of daptomycin for the MRSA isolates collected from the patients with previous anti-MRSA drug treatment was $1.05 \pm 1.13$, which was significantly higher than that for the MRSA isolates collected from the patients who had not been treated with anti-MRSA drugs $(0.39 \pm 0.27, p<0.0001)$. In the CRBSI group, the mean MIC of daptomycin for the MRSA isolates collected from the patients with previous antiMRSA drug treatment was $1.27 \pm 1.22$, which was significantly higher than that for the MRSA isolates collected from the patients who had not been treated with anti-MRSA drugs $(0.53 \pm 0.37, p<0.01)$. However, in the non-CRBSI group no significant difference in the mean MIC of daptomycin was observed between the MRSA isolates collected from the patients with and without a history of anti-MRSA drug treatment $(0.35 \pm 0.14$ vs. $0.32 \pm 0.18, p>0.05)$.

There was no difference in the mean MIC of vancomycin between the isolates collected from the CRBSI and non-CRBSI groups (1.18 \pm 0.41 vs. $1.13 \pm 0.4, p>0.05)$ (Figure $1 \mathrm{~b}$ ). The mean MIC of vancomycin for the 
isolates collected from the patients who had a history of anti-MRSA drug treatment was $1.33 \pm 0.48$, which was significantly higher than that for the isolates collected from the patients who had not been treated with anti-MRSA drugs $(1.11 \pm 0.37, \mathrm{p}<0.05)$. In the CRBSI group, the mean MIC of vancomycin for the isolates collected from the patients who had a history of anti-MRSA drug treatment was $1.38 \pm 0.5$, which was significantly higher than that for the isolates collected from the patients who had not been treated with anti-MRSA drugs $(1.08 \pm 0.32, p<0.05)$. However, in the non-CRBSI group no significant difference in the mean MIC of vancomycin was observed between the isolates collected from the patients with and without a history of anti-MRSA drug treatment $(1.2 \pm 0.45$ vs. $1.13 \pm 0.4, p>0.05)$.

In the multiple regression analysis, a significant CRBSI and a history of anti-MRSA drug treatment ( $p=0.0036$ and $p=0.0002$, respectively) were identified as factors related to the MIC of daptomycin (Table 2). Only a history of anti-MRSA drug treatment was found to be related to the MIC of vancomycin $(p=0.0302)$.

\section{Fluctuations in the MIC of daptomycin due to anti-MRSA drug treatment}

During the anti-MRSA drug treatment, MRSA was isolated again from the blood cultures of $10 \mathrm{CRBSI}$ patients (Figure 2a) and 4 non-CRBSI patients (Figure 2b). The anti-MRSA drugs administered to the CRBSI patients were daptomycin in 5 patients, vancomycin in 3 patients, and teicoplanin and linezolid in 1 patient each, whereas those administered to the non-CRBSI patients were teicoplanin in 3 patients and vancomycin in 1 patient. In both groups, each drug was administered at the standard dose. In the CRBSI group, the infected central venous catheters were not removed in the period between the blood cultures since the CRBSI had not been diagnosed at that time.

After treatment, all of the isolates collected from the CRBSI patients exhibited 1-2 log2 increases in the MIC of daptomycin. Four of them also demonstrated increases in the MIC of vancomycin. In one nonCRBSI case, only the MIC of daptomycin was increased, and in another case only the MIC of vancomycin was increased. No change in the MIC of either drug was observed in the other two cases. Among the CRBSI patients, the mean duration of anti-MRSA drug treatment was 18.3 days (11-31 days) for the 4 patients whose MRSA isolates exhibited increases in the MIC of both daptomycin and vancomycin and 25.5 days (7-61 days) for the 6 patients whose MRSA isolates only demonstrated increases in the MIC of daptomycin. In the non-CRBSI group, the patients whose isolates exhibited increases in the MIC of daptomycin and vancomycin were administered anti-MRSA drug treatment for 39 and 36 days, respectively. The two patients whose isolates did not exhibit changes in the MIC of either drug were administered anti-MRSA drug treatment for 8 and 15 days, respectively.

The relationships between the duration of anti-MRSA drug treatment and increases in the MIC of daptomycin or vancomycin are shown in Figure 3. In the CRBSI group, increases in the MIC of daptomycin were seen in 6 cases in which anti-MRSA drug treatment was administered for $\leq 20$ days and 3 cases in which anti-MRSA drug treatment was administered for 21-40 days, but in the non-CRBSI group an elevated MIC of daptomycin was only observed in 1 case, in which anti-MRSA drug treatment was administered for 21-40 days (Figure 3a). On the other hand, regarding the relationship between the 
duration of anti-MRSA drug treatment and the MIC of vancomycin, in the CRBSI group elevated MIC were seen in 3 cases in which anti-MRSA drug treatment was administered for $\leq 20$ days and 1 case in which anti-MRSA drug treatment was administered for 21-40 days, but in the non-CRBSI group an increased MIC was only seen in 1 case, in which anti-MRSA drug treatment was administered for 21-40 days (Figure 3b).

\section{Discussion}

In this study, we revealed that MRSA isolated from the blood of CRBSI patients had a higher MIC of daptomycin than that collected from the blood of non-CRBSI patients, especially among patients with a history of anti-MRSA drug treatment. Among the cases in which the MIC of daptomycin could be followed, the MIC of daptomycin increased in all of those involving CRBSI in which anti-MRSA drug treatment was administered for $\leq 20$ days at the standard dose.

The factors associated with the appearance of reduced susceptibility to daptomycin have been reported to include high bacterial loads derived from refractory infections, inadequate doses of daptomycin, and the absence of necessary surgical procedures [11]. These factors may be associated with reduced susceptibility to daptomycin in endocarditis [7] and osteomyelitis [8]. CRBSI may also be affected in similar ways, and if MRSA in a catheter biofilm is exposed to an anti-MRSA drug, strains with reduced susceptibility to daptomycin may survive and disperse in the blood.

As for the mechanism responsible for reduced susceptibility to daptomycin, repulsive force associated with an increase in the positive charge of the cell membrane has been suggested [12]. Alternatively, the mechanism may involve a gene mutation or non-gene mutation-related physiological changes in bacteria. $m p r F$ mutations are the most common gene mutations associated with reduced susceptibility to daptomycin, and it is said that they also produce cross resistance to vancomycin [13]. In this study, since many of the CRBSI-related isolates did not show an increase in the MIC of vancomycin, such mutations were considered to be irrelevant.

As for physiological changes, it has been reported that the bacteria in biofilms become less sensitive to antibiotics by slowing their growth rate [14]. It was also reported that when bacteria were exposed to low concentrations of antibiotics, their cell walls thickened, and their susceptibility to antibiotics reduced, and when they were transferred to a drug-free medium the thickness of their cell walls normalized [15]. Furthermore, the cell walls of MRSA have been reported to thicken after the administration of daptomycin [16]. Therefore, it is considered that when MRSA biofilms form on medical devices the thickness of the cell walls of the bacteria increases, which makes it difficult for daptomycin to reach the cell membrane, resulting in an increased MIC.

Although the mechanisms responsible for reduced susceptibility to daptomycin could not be elucidated in this study, clinical measures need to be put in place to prevent the emergence of CRBSI. It is of course important to prevent the occurrence of CRBSI by employing maximal barrier precautions when inserting catheters and by using catheters made of materials that are not amenable to biofilm formation, such as polyurethane. If a CRBSI due to MRSA is suspected, it is necessary to remove the infected catheter 
immediately. If the catheter cannot be removed immediately, high-dose daptomycin treatment should be considered as a way to reduce treatment tolerance and improve treatment efficacy, as it has been reported that high-dose daptomycin treatment is effective against endocarditis [17].

\section{Conclusions}

It is considered that when MRSA in catheter biofilms is exposed to anti-MRSA drugs, strains with reduced susceptibility to daptomycin are able to survive and disperse into the blood. Further study of whether high-dose daptomycin treatment is effective when catheters cannot be immediately removed is needed.

\section{Abbreviations}

CRBSI, catheter-related bloodstream infection; MIC, minimum inhibitory concentrations; MRSA, methicillin-resistant Staphylococcus aureus; VISA, vancomycin-intermediate Staphylococcus aureus; VRE, vancomycin-resistant Enterococcus

\section{Declarations}

\section{Authors' contributions}

SO, YM, KK designed the study. SO, TN, KM conducted the study and analyzed the data. SO, YM, KK wrote the paper. KK had primary responsibility for the final content. All authors read and approved the final manuscript.

\section{Funding}

No external founding was received.

\section{Availability of data and materials}

The datasets used and/or analyzed during the current study are available from the corresponding author on reasonable request.

\section{Ethics approval and consent to participate}

This study was approved by the institutional review board of Teikyo University (committee number: 21031, approval date: May 24, 2021) and was conducted in accordance with the institutional guidelines.

\section{Consent for publication}

Not applicable.

\section{Competing interests}


The authors declare that they have no competing interests.

\section{References}

1. Wisplinghoff $H$, Bischoff T, Tallent SM, Seifert $H$, Wenzel RP, Edmond MB. Nosocomial bloodstream infections in US hospitals: analysis of 24,179 cases from a prospective nationwide surveillance study. Clin Infect Dis. 2004;39:309-17.

2. Matsushima A, Takakura S, Yamamoto M, Matsumura Y, Shirano M, Nagao M, et al. Regional spread and control of vancomycin-resistant Enterococcus faecium and Enterococcus faecalis in Kyoto, Japan. Eur J Clin Microbiol Infect Dis. 2012;31:1095-100.

3. Hanaki H, Cui L, Ikeda-Dantsuji Y, Nakae T, Honda J, Yanagihara K, et al. Antibiotic susceptibility survey of blood-borne MRSA isolates in Japan from 2008 through 2011. J Infect Chemother. 2014;20:527-34.

4. Esposito S, Purrello SM, Bonnet E, Novelli A, Tripodi F, Pascale R, et al. Central venous catheter-related biofilm infections: An up-to-date focus on meticillin-resistant Staphylococcus aureus. J Glob Antimicrob Resist. 2013;1:71-8.

5. Silverman JA, Perlmutter NG, Shapiro HM. Correlation of daptomycin bactericidal activity and membrane depolarization in Staphylococcus aureus. Antimicrob Agents Chemother. 2003;47:253844.

6. Sakoulas G, Alder J, Thauvin-Eliopoulos C, Moellering RC Jr, Eliopoulos GM. Induction of daptomycin heterogeneous susceptibility in Staphylococcus aureus by exposure to vancomycin. Antimicrob Agents Chemother. 2006;50:1581-5.

7. Ji S, Jiang S, Wei X, Sun L, Wang H, Zhao F, et al. In-Host Evolution of Daptomycin resistance and heteroresistance in methicillin-resistant Staphylococcus aureus strains from three endocarditis patients. J Infect Dis. 2020;221:243-52.

8. van Hal SJ, Paterson DL, Gosbell IB. Emergence of daptomycin resistance following vancomycinunresponsive Staphylococcus aureus bacteraemia in a daptomycin-naïve patient-a review of the literature. Eur J Clin Microbiol Infect Dis. 2011;30:603-10.

9. Horan TC, Andrus M, Dudeck MA. CDC/NHSN surveillance definition of health care-associated infection and criteria for specific types of infections in the acute care setting. Am J Infect Control. 2008;36:309-32.

10. Brun-Buisson C, Abrouk F, Legrand P, Huet $Y$, Larabi S, Rapin M. Diagnosis of central venous catheterrelated sepsis. Critical level of quantitative tip cultures. Arch Intern Med. 1987;147:873-7.

11. Moise PA, North D, Steenbergen JN, Sakoulas G. Susceptibility relationship between vancomycin and daptomycin in Staphylococcus aureus: facts and assumptions. Lancet Infect Dis. 2009;9:617-24.

12. Heidary M, Khosravi AD, Khoshnood S, Nasiri MJ, Soleimani S, Goudarzi M. Daptomycin. J Antimicrob Chemother. 2018;73:1-11. 
13. Thitiananpakorn K, Aiba Y, Tan XE, Watanabe S, Kiga K, Sato'o Y, et al. Association of $m p r F$ mutations with cross-resistance to daptomycin and vancomycin in methicillin-resistant Staphylococcus aureus (MRSA). Sci Rep. 2020;10:16107.

14. Donlan RM, Costerton JW. Biofilms: survival mechanisms of clinically relevant microorganisms. Clin Microbiol Rev. 2002;15:167-93.

15. Lorian V, Atkinson B. Effects of subinhibitory concentrations of antibiotics on cross walls of cocci. Antimicrob Agents Chemother. 1976;9:1043-55.

16. Kanesaka I, Fujisaki S, Aiba Y, Watanabe S, Mikawa T, Katsuse AK, et al. Characterization of compensatory mutations associated with restoration of daptomycin-susceptibility in daptomycin non-susceptible methicillin-resistant Staphylococcus aureus and the role mprF mutations. J Infect Chemother. 2019;25:1-5.

17. Seaton RA, Gonzalez-Ruiz A, Cleveland KO, Couch KA, Pathan R, Hamed K. Real-world daptomycin use across wide geographical regions: results from a pooled analysis of CORE and EU-CORE. Ann Clin Microbiol Antimicrob. 2016;15:18.

\section{Tables}

Table 1. Characteristics of the patients

\begin{tabular}{lccc}
\hline & $\begin{array}{c}\text { Catheter-related } \\
\text { bloodstream infection }\end{array}$ & $\begin{array}{c}\text { Non-catheter-related } \\
\text { bloodstream infection }\end{array}$ & P value \\
\cline { 2 - 3 } & $\mathrm{n}=46$ & $\mathrm{n}=69$ & \\
Mean age & 77.7 & 72.2 & 0.06 \\
$\begin{array}{l}\text { Sex } \\
\text { Female }\end{array}$ & 24 & & \\
$\begin{array}{l}\text { Male } \\
\text { Prior exposure to }\end{array}$ & 22 & 24 & 0.08 \\
anti-MRSA drug & 16 & 45 & 0.0003 \\
\hline
\end{tabular}


Table 2. Multiple regression analysis of the minimum inhibitory concentrations

Catheter-related bloodstream infection

0.3131

Daptomycin

\begin{tabular}{lll}
\hline $\begin{array}{c}\text { Parameter } \\
\text { Estimate }\end{array}$ & $95 \% \mathrm{Cl}$ & P value
\end{tabular}

Vancomycin

\begin{tabular}{lll}
\hline $\begin{array}{c}\text { Parameter } \\
\text { Estimate }\end{array}$ & $95 \% \mathrm{Cl} \quad$ P value
\end{tabular}

Prior exposure to anti-MRSA drug

0.5207

0.2564 to 0.7849

0.0002

0.2250

0.0219 to 0.42810 .0302

Figures

a

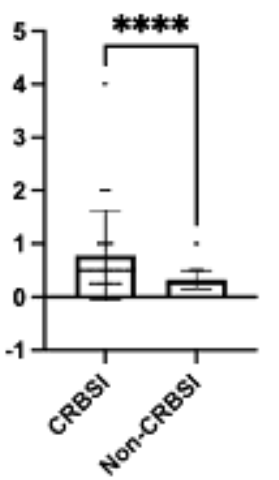

DAP-MIC CRBSI+ aMRSAdrug+,-

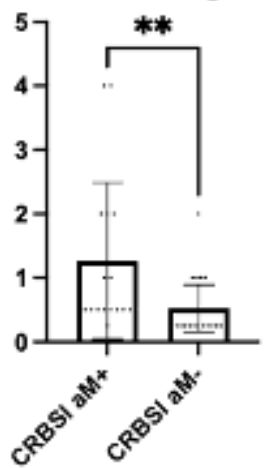

DAP-MIC aMRSAdrug+,-

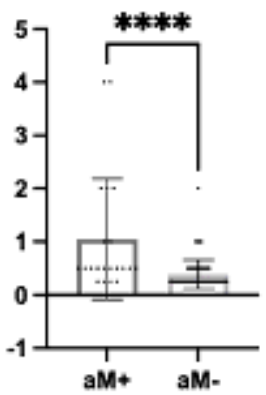

DAP-MIC CRBSIaMRSAdrug+,-

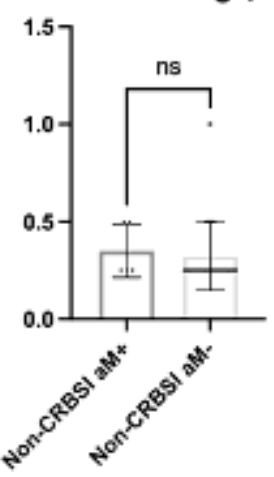

b

VCM-MIC CRBSI+,-

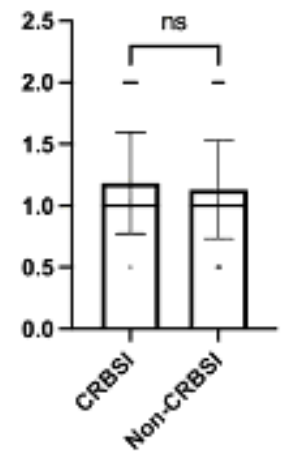

VCM-MIC CRBSI+_ aMRSAdrug+,-

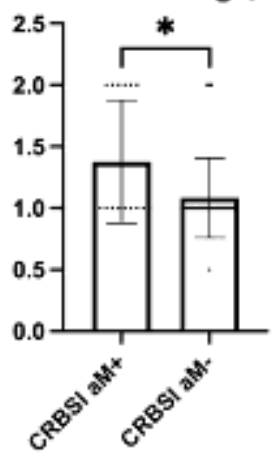

VCM-MIC aMRSAdrug+,-

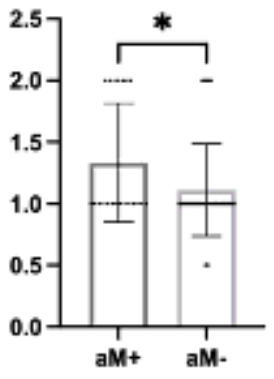

VCM-MIC CRBSIaMRSAdrug+,-

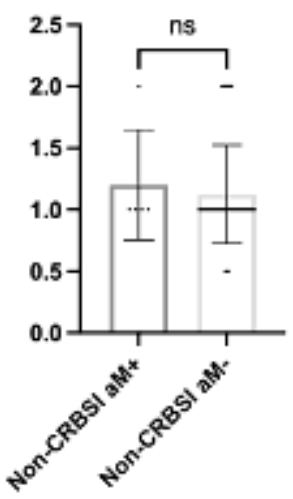

Figure 1

Figure 1 
The minimum inhibitory concentrations of daptomycin (a) and vancomycin (b) for isolates collected from CRBSI or non-CRBSI patients with or without a history of anti-MRSA drug treatment. DAP, daptomycin; VCM, vancomycin; MIC, minimum inhibitory concentration; CRBSI, catheter-related bloodstream infection; aM, anti-MRSA drug treatment; ${ }^{*} p<0.05,{ }^{\star *} p<0.01,{ }^{\star \star \star *} \mathrm{p}<0.0001$

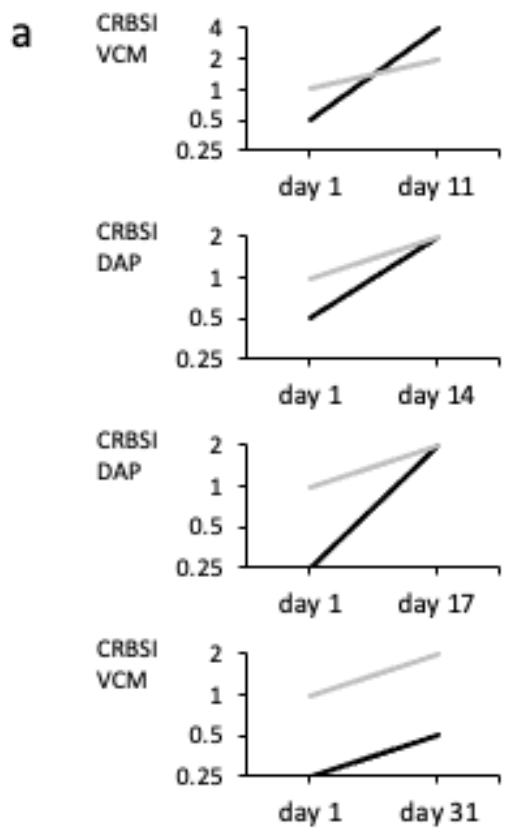

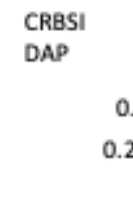

$$
\begin{aligned}
& \text { CRBSI } \\
& \text { TEIC }
\end{aligned}
$$
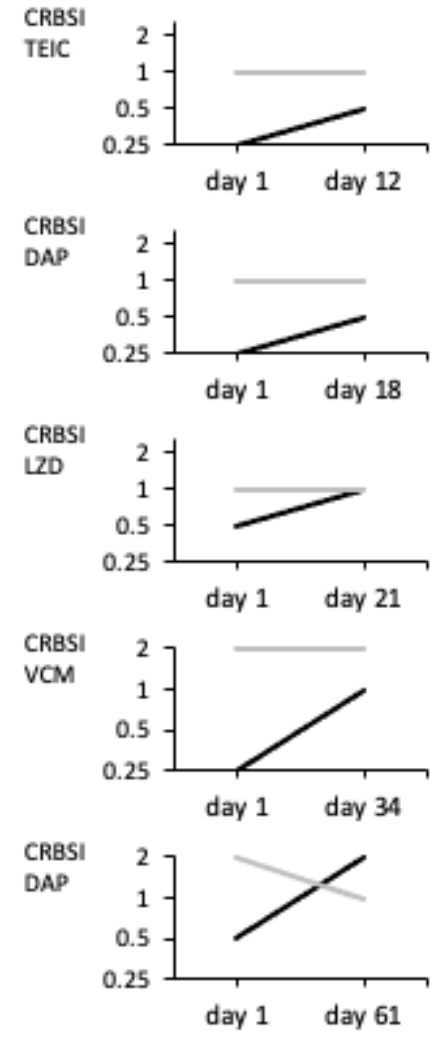

Figure 2
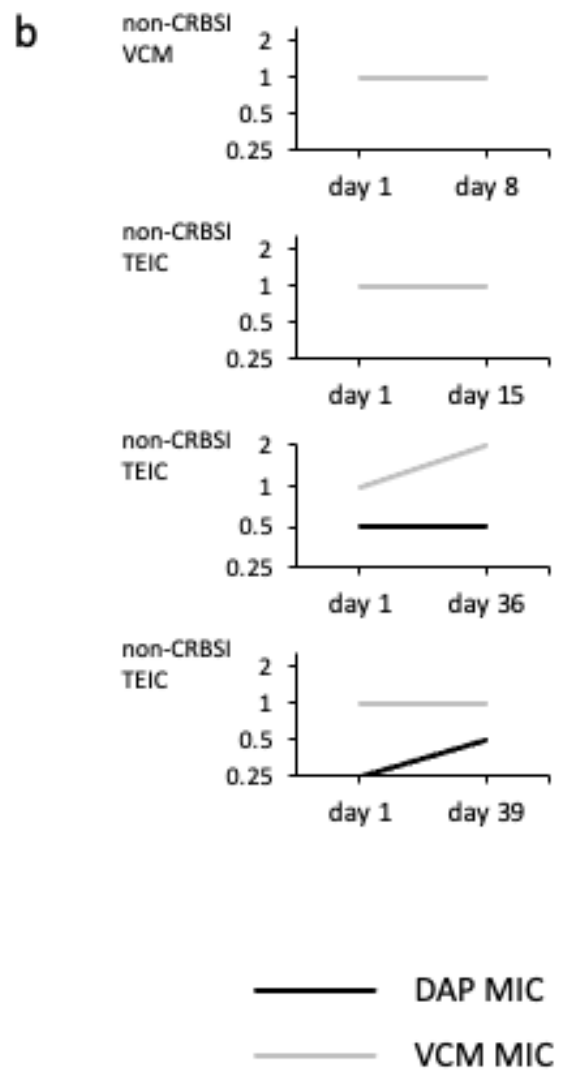

\section{Figure 2}

Fluctuations in the MIC of daptomycin due to anti-MRSA drug treatment. During the anti-MRSA drug treatment, MRSA was isolated again from the blood cultures of $10 \mathrm{CRBSI}$ patients (a) and 4 non-CRBSI patients (b). CRBSI, catheter-related bloodstream infection; VCM, vancomycin; DAP, daptomycin; TEIC, teicoplanin; LZD, linezolid 
a

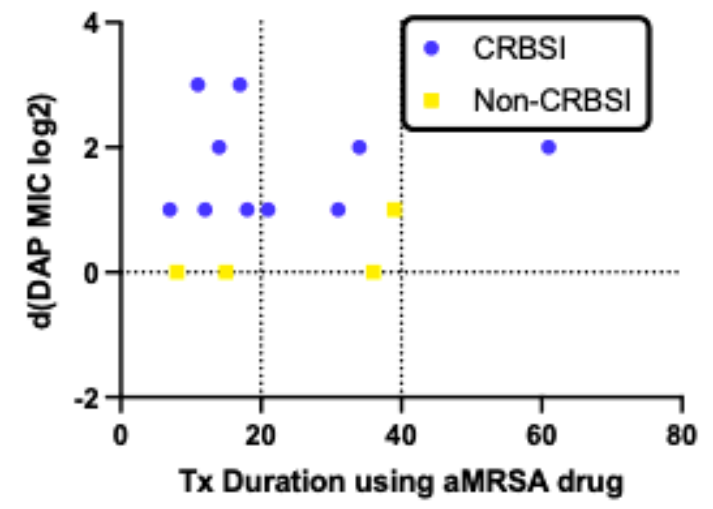

b

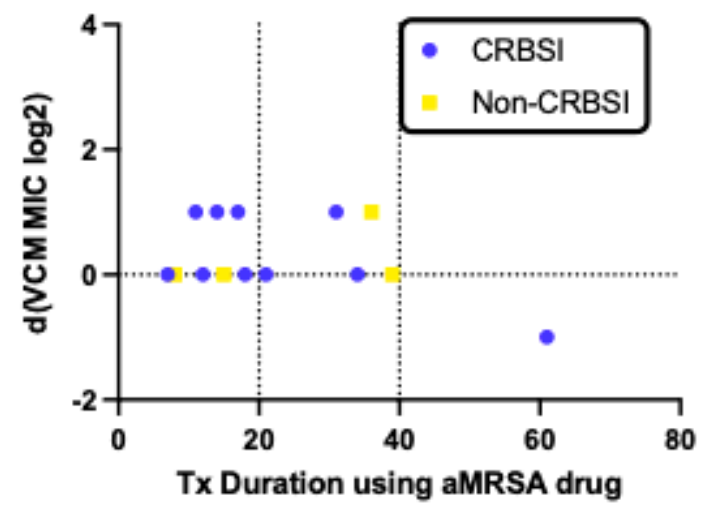

Figure 3

\section{Figure 3}

Relationships between the duration of anti-MRSA drug treatment and increases in the MIC of daptomycin (a) or vancomycin (b). Blue circles and yellow squares indicate CRBSI and non-CRBSI cases, respectively. 